\title{
Evolución de la equidad interterritorial en el acceso a los servicios sanitarios entre las CCAA españolas (1987-2003) y su relación con la evolución del gasto sanitario público
}

\author{
Montero Granados, Roberto \\ Jiménez Aguilera, Juan de Dios \\ Universidad de Granada
}

\begin{abstract}
Resumen:
El debate sobre la equidad interterritorial en el acceso a los servicios públicos básicos está permanentemente abierto en España. En el caso de la sanidad, la generalización de las competencias sobre su gestión a las Comunidades Autónomas (2002), unido al crecimiento y la heterogeneidad en el gasto sanitario que ello ha comportado puede estar incidiendo en la igualdad de acceso a los servicios. En este artículo, a partir de las Encuestas nacionales de salud, se estudia la equidad de acceso a cinco servicios sanitarios básicos desde 1987 a 2003. Los resultados apuntan a que durante el período analizado se producen importantes desigualdades en el acceso a los distintos servicios sanitarios en función de la CA de residencia y que dicha desigualdad no se está reduciendo con el tiempo. También se ha obtenido una correlación significativa entre la desigualdad de acceso a los servicios sanitarios y el gasto sanitario en cada CA en aquellos servicios sanitarios cuya decisión de acceso depende exclusivamente del paciente, aunque dicha correlación desaparece cuando los servicios deben ser recomendados por algún facultativo.
\end{abstract}

Palabras clave: equidad interterritorial, gasto sanitario, descentralización, equidad de acceso. Códigos JEL: D63; H23; H51; H43; H73; I18.

\section{Resumen:}

The debate about territorial equity access to the basic public services is permanently open in Spain. In the case of the sanity public services, the assumption of responsibility by the Autonomous Communities (AC) (2002), together with the growth and the heterogeneity in the sanitary public service expenses can to have effects in the access equality to the services. In this article, with the national Surveys of health data, we studied the access to five basic sanitary services from 1987 to 2003. The results point to, during the analyzed period, important inequalities take place in the access to the different sanitary services in function of the $A C$ residence and that this inequality is not decreasing with the time. A significant correlation has also been obtained among the access inequality to the publics sanitary services and the sanitary expense in each $A C$ in those sanitary services whose access decision depends exclusively on the patient, although this correlation disappears when the services should be recommended by a physician.

Key words: territorial equity, sanitary public services expenditure, decentralization, equity in publics services access.

JEL Classification: D63; H23; H51; H43; H73; I18. 


\section{Introducción}

De los motivos de intervención pública que enumera Musgrave (1959) —eficiencia, equidad y optimización macroeconómica — quizá sea el de la equidad el que genere una mayor controversia. Por lo general, existe un amplio acuerdo sobre la bondad de las políticas que ayuden a la eficiencia de los mercados, tanto a su perfeccionamiento como a su ampliación, y siempre que se establezca el correspondiente vínculo entre política y eficiencia son siempre bien recibidas. Asimismo, existe también un amplio consenso sobre la necesidad de políticas macroeconómicas de estabilización, para contrarrestar los grandes ciclos, a favor del crecimiento a largo plazo, de forma que prácticamente nadie discute la efectividad de dichas políticas.

Sin embargo, a pesar de que desde una perspectiva práctica existe una amplia unanimidad en considerar como negativas algunas situaciones de inequidad, en particular que la distribución de la renta puede ser muy injusta con algunos individuos, así como en la bondad de establecer algún criterio normativo para alcanzar algún objetivo ético de redistribución, la concreción de dicho objetivo ético, es decir de la cantidad o calidad de redistribución que se considera justa, no está exenta de controversia. En la mayor parte de los países democráticos, posiblemente por la influencia de motivos de tipo político, la justicia social se vincula con la idea de equidad, de igualdad. Es decir, se considera que un sistema redistributivo es justo en la medida que promueva la equidad. Las teorías rawlsianas (Rawls, J., 1971) sobre la justicia social aportan cierta cobertura teórica a dicha vinculación, lo cual no quiere decir, ni que dicho criterio de justicia sea el único o que sea el mejor posible. Así las nuevas teorías utilitaristas (Harsanyi, 1955) y las neoliberales (Nozick, 1974; Hayek, 1976; Sandel, 1982) continúan ofreciendo ideas alternativas sobre los límites a la redistribución. Por su parte, algunos autores (Buchanan, 1975; Lindbeck y Weibull, 1988; Pedersen, 2001), alrededor del denominado «dilema del samaritano», comienzan a dudar de la capacidad de algunas políticas redistributivas para reducir realmente la desigualdad.

A pesar de esta controversia, algún objetivo de reducción de la desigualdad debe impulsar la intervención pública. La existencia misma de un sector público y el tamaño relativo que está alcanzando el sector público en la actualidad en las economías más desarrolladas no puede entenderse sin referirse a este objetivo normativo. En este sentido, la mayor parte de los servicios públicos tienen un importante papel redistributivo (Alesina et al., 1999; Atkinson y Stiglitz, 1988; Jha, 1998; Tanzi, 2000). Siempre que sean sufragados por impuestos progresivos o, como mínimo, proporcionales a la renta y siempre que puedan ser utilizados de forma igualitaria se obtendrá un vector redistributivo que se ajusta a los criterios normativos de redistribución.

El caso de los servicios sanitarios públicos es especialmente relevante. Estos disponen, en España, un presupuesto consolidado que, en $2003^{1}$, alcanzó los 41,199.7 millones de euros, lo que supone 903 euros por habitante y un 5,49\% del PIB. Cifras

Estadísticas del gasto sanitario público (2004). 
ligeramente inferiores a las de los países de referencia. Para la Unión Europea (UE), la media de gasto públicos sanitario fue, para el mismo año ${ }^{2}$, del 6,8\% del PIB. El potencial redistributivo de tan importante cantidad de recursos no puede pasar desapercibido ni debe quedar desaprovechado. En efecto, el artículo 12 de la Ley General de Sanidad $^{3}$ (LGS) establece que «los poderes públicos orientarán sus políticas de gasto sanitario en orden a corregir desigualdades sanitarias y garantizar la igualdad de acceso a los servicios sanitarios públicos en todo el territorio español» aludiendo explícitamente a las directrices de los artículos 9.2 y 158.1 de la Constitución Española (CE). En este último artículo de la CE se afirma expresamente que «En los Presupuestos Generales del Estado podrá establecerse una asignación a las Comunidades Autónomas en función del volumen de los servicios y actividades estatales que hayan asumido y de la garantía de un nivel mínimo en la prestación de los servicios públicos fundamentales en todo el territorio español.» Ambas referencias comportan cuando menos dos implicaciones para la política pública: a) una reiteración de la importancia de la equidad en el acceso, independientemente de la residencia; y b) la existencia de unos servicios públicos como fundamentales en todo el territorio español, entre los que se encuentran incluidos los servicios sanitarios.

La equidad en el acceso al servicio público puede ser estudiada desde múltiples puntos de vista: horizontal, vertical, en función de la renta, igualdad de oportunidades, etc. En el caso español, sin embargo, hemos visto como el legislador ha tratado de resaltar especialmente la vertiente geográfica. En este sentido, la equidad se define como igualdad de acceso a los servicios sanitarios en todo el territorio español independientemente de la CA de residencia, y debe garantizarse, si es necesario, incluso con la dotación de recursos adicionales especiales. Esta especial preocupación no es trivial, sino que adquiere plena justificación en un entorno de descentralización de la gestión del servicio sanitario que promueve el actual sistema de administración español, cuasi-federal, que ha venido en denominarse como estado de las autonomías.

La descentralización sanitaria en España puede estudiarse desde muchos ángulos distintos y cada uno de ellos dará origen a una clasificación o periodificación distinta. Una buena revisión se recoge en Cabases (ed.) et al., 2006). Desde una perspectiva temporal, en 1981 comienza el proceso con la cesión de competencias a la Comunidad Autónoma (CA) de Cataluña, que posteriormente se completaría con Andalucía (1984), Valencia y País Vasco (1988), Galicia y Navarra (1991) y finalmente Canarias (1994), completando el traspaso a las autonomías denominadas «del $151 »{ }^{4}$. Este periodo se caracteriza por el desarrollo de complejas negociaciones bilaterales para acordar el volumen de recursos necesario y por la implantación de heterogéneos sistemas de financiación que, por no gozar de la transparencia o justificación mínima

OECD Health Data (Ministerio de Salud y Consumo, 2004)

Ley $14 / 86$ de 25 de abril.

4 En alusión al artículo 151 de la CE que permitía a estas CCAA, por disponerlo en sus respectivos estatutos de autonomía, una más rápida adquisición de competencias. 
que deben exigir a u sistema de distribución de recursos públicos, han sido muy criticados (Sánchez y Gómez, 1998; López, 1998; López y Rodrigo, 2000). El proceso de traspaso de competencias no se completaría hasta 2002 en que acceden a la gestión sanitaria el resto de Comunidades autónomas (CCAA). Proceso que desemboca en nuevas negociaciones sobre el coste del servicio para las CCAA del hasta entonces denominado territorio INSALUD, y un nuevo sistema de financiación, que contiene casi tantas garantías y restricciones como CCAA, de forma que la financiación obtenida y el gasto por cada CA son muy heterogéneos. En un reciente estudio del Instituto de Estudios Fiscales (IEF, 2005), se pone de manifiesto que en 2003 la media de gasto sanitario público total en España fue de 953 euros por persona protegida ${ }^{5}$, lo que no impide el que se produzca un amplio rango de gasto entre CCAA que oscila entre 870 euros/persona protegida (Madrid) y los 1084 euros/persona protegida (Navarra). Dispersión que, según la misma fuente (IEF, 2005, p. 157), ha tendido a crecer desde 2000.

La creciente dispersión del gasto sanitario público puede estar afectando a la equidad en el acceso a los servicios sanitarios públicos, con las consecuencias de índole ético que ésta desigualdad podría dar lugar (Rice y Smith, 2001). En este sentido, el objetivo del presente artículo es medir la dispersión en el acceso a los servicios sanitarios públicos y su relación con el gasto público. Más concretamente, trataremos de medir si un individuo, con unas determinadas características personales y sanitarias, tiene la misma probabilidad de recibir una asistencia sanitaria independientemente de la CA de residencia. Si no existen diferencias significativas entre la probabilidad de uso de un servicio sanitario en función de la CA de residencia podremos concluir que se cumple el principio de equidad en el acceso y viceversa. Sin embargo, la equidad en el acceso no debe medirse sólo de forma estática, sino que también debe considerarse si dicha equidad está variando (aumentando o disminuyendo) con el tiempo. Es decir, el hecho de que, en un momento determinado se esté produciendo una determinada inequidad no es justificación suficiente para juzgar como negativa o positiva una determinada política, sino que será necesario evaluar su impacto a lo largo del tiempo, observando si dicha inequidad tiende a reducirse o a ampliarse. El estudio del uso sanitario se llevará a efecto mediante el uso de las Encuestas Nacionales de Salud desde 1987 hasta 2003 (última disponible hasta la fecha), publicadas por el Instituto Nacional de Estadística (INE).

Debemos hacer énfasis de que, en este trabajo, se está identificando equidad en el acceso con equidad en el uso, debido a que los datos de que se disponen son de uso. Sin embargo, haciéndonos eco de una delicada controversia (Mooney, 1983; Mooney et at., 1991a y 1991b; Culyer et al., 1992a y 1992b), conviene puntualizar algunas de las limitaciones más importantes que este enfoque representa. En principio, acceso no es uso principalmente por dos motivos: a) desde el punto de vista de la demanda, porque hemos de considerar el principio de libre albedrío y libertad de cada individuo de usar

La población protegida es la población de derecho de cada CA de la que se detraen los colectivos acogidos a entidades especiales de la Seguridad Social como MUFACE, MUJEJU, ISFAS etc. 
o no un servicio público. Por ejemplo, dos individuos con igualdad de acceso e iguales características personales pueden decidir libremente usar (uno) y no usar (otro) el servicio público y ello no constituiría ninguna inequidad; $\mathrm{y} b$ ) desde el punto de vista de la oferta, porque hemos de suponer la existencia de variabilidad en la práctica clínica y la libertad de cada facultativo de decidir, de buena fe, cual es el mejor tratamiento de entre varios alternativos, en el que ninguno es dominante, por ejemplo, dos facultativos pueden decidir tratamiento y no tratamiento a dos pacientes con las mismas características y ello no comportaría ninguna inequidad del servicio sanitario.

Sin embargo, aunque no es descabellado pensar que es posible que algunas personas utilicen los servicios sanitarios menos de lo que deberían y otras más, ello no implica necesariamente que la media esté sesgada. El mismo criterio puede adoptarse por parte de la variabilidad en la práctica; es decir, el exceso de tratamiento recomendado por un facultativo puede compensarse con el defecto de tratamiento por parte de otro, de forma que dos fenómenos que pueden ser fuente de grandes diferencias a nivel individual se convierten en un problema irrelevante a nivel agregado, que se puede solucionar, al menos en parte, utilizando métodos probabilísticos de estimación que contemplen la posibilidad de sobre o infradispersión.

Por otro lado, mientras que son relativamente frecuentes los trabajos que relacionan salud con territorio (Rosenberg y Wilson, 2000; Haynes, 1991; Stern, 1983), no son muchos los que relacionan específicamente uso y territorio de residencia (Goddart y Smith, 2001; Birch y Abelson, 1993; Watt et al., 1994). Quizás la razón sea los problemas de concreción teórica que menciona Rice y Smith $(2001)^{6}$, Aunque todos reconocen la relación, sus consideraciones no suelen pasar de ser meramente teóricas o, en el caso del último autor, de limitarse a comparar territorios rurales con urbanos. Para el caso español, la ausencia de trabajos es prácticamente total si exceptuamos los trabajos de Benach y Yasui (1999) y de Borell et al. (1999), aunque ambas se refieren a la relación entre salud y territorio, y en este último, además, sólo alude a casos de mortalidad en Madrid y Barcelona. En este sentido, esta investigación trata de llenar un hueco en la literatura mediante la elaboración de un indicador directo y robusto de la relación entre acceso y territorio aplicada al caso español.

En concreto, nuestro objetivo es medir la utilización estandarizada en cada CA española, desde 1987 hasta 2003, de cinco servicios sanitarios públicos: a) consumo de medicamentos con receta; b) visitas al médico generalista; c) visitas al médico especialista; d) uso del servicio de urgencias; y e) días de hospitalización ${ }^{7}$, en el sentido de comprobar en que medida el uso de dichos servicios es o no heterogéneo, así

6 Se lamentan especialmente de la ausencia de una teoría base y una falta de un criterio claro entre los autores para discriminar entre los efectos de la oferta y la demanda.

7 Se han escogido dichas dimensiones porque son las que constan de forma sistemática y comparable en todas las encuestas del periodo. En 2003 también pregunta por el consumo de medicina alternativa. En todas se pregunta por la asistencia de dentista pero este consumo es muy mayoritariamente privado, aunque en algunas CCAA está provisto públicamente hasta determinadas edades. Finalmente en la encuesta de 1999 también se pregunta por muchas mas dimensiones de consumo (sobre todo especialidades, ambulancia y pruebas diagnósticas) 
como su evolución en el tiempo. En una segunda aproximación dado que el interés de esta investigación es observar si la diversidad en la política de descentralización puede incidir en la equidad, se comprobará la correlación entre uso de servicios sanitarios y gasto sanitario.

\section{Metodología}

Se han obtenido los datos individuales de las 7 oleadas de la Encuesta Nacional de Salud que el Ministerio de Sanidad y Consumo (MSC) y el Instituto Nacional de Estadística (INE) han publicado hasta la fecha, que corresponden a los años 1987-19931995-1997-1999-2001 y 2003. Constituyen u total de 166,108 observaciones de unas encuestas realizadas a mayores de 15 años, por el procedimiento de muestreo estratificado, en que, entre otros asuntos de relevancia, se pregunta a los encuestados sobre su residencia, su estado de salud y el uso que hacen de distintos servicios sanitarios. De dichas encuestas se han seleccionado las siguientes variables independientes:

- ca: CA de residencia, que se ha transformado en diecisiete dummies dicotómicas regionales, una por CA. Estas serán las variables de interés en este estudio. Por su baja población y especiales condiciones sanitarias se han excluido las ciudades autónomas de Ceuta y Melilla.

- edad_m: la encuesta recoge la edad del encuestado que, mediante una transformación lineal, se ha convertido en una variable relativa a la edad medida de la encuesta. Dicha transformación es necesaria en orden a conocer la probabilidad de ocurrencia del sujeto de referencia.

- género: variable dicotómica que adopta los valores 0 mujer y 1 varón.

- estasalud: variable que puede adoptar hasta cinco valores desde 1: muy buena salud; a 5: muy mala salud. Se han elaborado 5 variables dummies dicotómicas

— sum_enf: suma de las enfermedades crónicas de que ha sido diagnosticado el sujeto entrevistado.

- factor: factor de elevación de la observación al total poblacional. Este no estaba disponible en las encuestas de 1993, 1995 y 1997.

La selección de dichas variables responde a la necesidad de estandarizar indirectamente la probabilidad de uso en cada CA. Se utilizan factores demográficos (edad, género) y de salud, tales como la autovaloración (subjetiva) y las enfermedades diagnosticadas (objetiva). La utilización de éstas variables en la literatura está prácticamente generalizada, por lo que no debería requerir de mayor justificación (Urbanos, 2000; Kakwani et al., 1997; Alvarez, 2001; Jiménez et al., 2002; entre otros)

En el modelo utilizado, las variables dependientes serán las relativas al uso de los siguientes servicios sanitarios públicos, que en la encuesta tienen las siguientes definiciones: 
- farmacia: días en que ha consumido medicamentos con receta del Sistema Nacional de Salud (SNS) en las dos semanas inmediatamente anteriores a la encuesta.

- medprim: visita al médico generalista (excluidos los odontólogos y para pedir cita) del SNS en las dos semanas inmediatamente anteriores a la encuesta.

- medespe: visita al médico especialista del SNS en las dos semanas inmediatamente anteriores a la encuesta.

- urgencias: atención en un servicio público de urgencias, incluida la asistencia a domicilio, durante un año, desde la fecha de la encuesta.

- hospital: días de ingreso en un hospital público durante un año, desde la fecha de la encuesta.

Como en todos los estudios basados en encuestas hemos de reconocer las limitaciones usuales de sesgo en la selección de la muestra, en las opiniones vertidas, en la posible incorrelaciones de causa-efecto, por haberse realizado en fechas distintas que pudieran tener efectos estacionales, etc. Además, como advierte Urbanos (2000) dicha encuesta se realiza sobre población no institucionalizada ${ }^{8}$. Dichas limitaciones pueden sesgar los resultados, aunque de forma indeterminada y en menor medida cuanto mayor sea el tamaño muestral. Paralelamente, se han presentado algunos otros problemas metodológicos, como:

- Integración de la macro encuesta de 1999 con el resto de encuestas debido a que aquella tenía un cuestionario relativamente distinto. Por ejemplo, en el caso del consumo de medicamentos sólo se pregunta si se ha consumido o no en los últimos 14 días y no por cuantos días se ha consumido. Tampoco se pregunta por las visitas a urgencias y no se distingue entre visitas a médicos generalistas y especialistas. A falta de algún criterio de discriminación se ha considerado que las visitas al médico lo eran al médico generalista, lo cual puede haber sesgado la estimación para ese año de esa variable.

- Debido al escaso tamaño de la muestra, en 1997 no han convergido las estimaciones de recuento truncadas para las variables medprim y medespe.

- Las encuestas de 1993, 1995 y 1997 no incluyen ningún campo como factor de elevación de cada observación de la muestra a la población lo que puede provocar algún sesgo si el muestreo no ha sido realizado de una forma suficientemente aleatoria.

El Cuadro 1 recoge algunos estadísticos descriptivos de las variables dependientes. Respecto al género se aprecia que los varones son minoría (un 48\%). respecto a la edad se observa un progresivo envejecimiento de la encuesta. No se aprecia mejoría general en el estado de salud, ni como autovaloración del estado de salud ni como

8 Individuos que no están internados en una institución pública como hospitales, cárceles o cuarteles. 


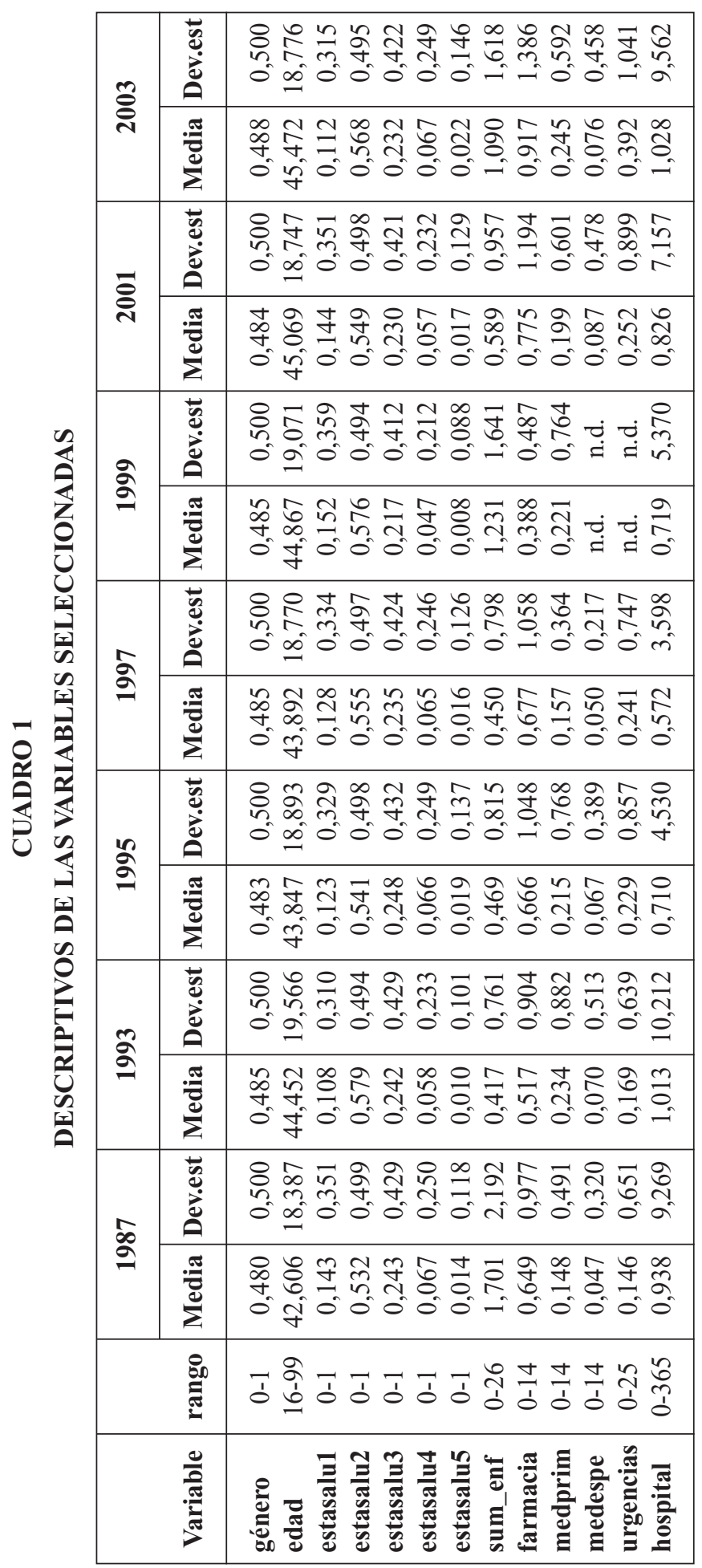


suma de enfermedades. Finalmente, salvo el caso de asistencia hospitalaria, se observa que se está produciendo un incremento generalizado del uso, sobre todo a partir de 1999.

En el cuadro 2 se presentan el número de observaciones correspondientes a cada CA en cada encuesta. El bajo número de encuestas de 1995 y 1997 obligan a considerar los resultados de estos años con las debidas cautelas.

Para la estimación de la probabilidad de uso estandarizada de cada uno de los cinco servicios sanitarios se utilizará un modelo, usual en la literatura, de dos partes o de valla. El modelo supone que existen dos procesos independientes en el consumo sanitario, uno primero que depende del paciente, que puede decidir o no acudir a la consulta, y un segundo que depende del facultativo que puede decidir el tratamiento o las sucesivas consultas o consumos de servicio sanitario. Un modelo de valla es «un modelo de recuento modificado en el que los dos procesos, los que generan ceros y los que generan valores positivos no tienen porqué ser los mismos» (Cameron y Trivedi, 1998) En este modelo se realizan, por tanto dos estimaciones de los parámetros, los que indican si se acude o no a un servicio y los que indican cuanto servicio se consume una vez que se ha decidido acudir por primera vez. En la forma:

$$
P\left(Y_{i, j} \mid X_{i}, R_{i}\right)=F_{j}\left(R_{i} ; X_{i} ; B_{j}\left(R_{i} ; X_{i}\right)\right)
$$

\section{CUADRO 2}

NÚMERO DE OBSERVACIONES POR CC.AA.

\begin{tabular}{|l|r|r|r|r|r|r|r|}
\hline \multicolumn{1}{|c|}{ CCAA } & $\mathbf{1 9 8 7}$ & $\mathbf{1 9 9 3}$ & $\mathbf{1 9 9 5}$ & $\mathbf{1 9 9 7}$ & $\mathbf{1 9 9 9}$ & $\mathbf{2 0 0 1}$ & $\mathbf{2 0 0 3}$ \\
\hline Andalucía & 5.007 & 1.993 & 1.087 & 1.085 & 10.653 & 3.673 & 3.692 \\
Aragón & 967 & 1.003 & 204 & 204 & 1.846 & 634 & 630 \\
Asturias & 891 & 778 & 192 & 192 & 1.704 & 580 & 565 \\
Baleares & 545 & 799 & 128 & 128 & 1.149 & 385 & 462 \\
Canarias & 1.061 & 996 & 244 & 243 & 2.455 & 829 & 919 \\
Cantabria & 411 & 800 & 84 & 84 & 833 & 285 & 282 \\
Castilla-LM & 1.319 & 998 & 276 & 276 & 2.590 & 892 & 1.278 \\
Castilla-L & 2.088 & 1.501 & 432 & 432 & 3.891 & 1.350 & 903 \\
Cataluña & 4.681 & 1.997 & 1.024 & 1.024 & 9.553 & 3.238 & 3.354 \\
C. Valenciana & 2.875 & 1.502 & 620 & 620 & 6.101 & 2.078 & 2.207 \\
Extremadura & 848 & 1.000 & 176 & 176 & 1.610 & 557 & 528 \\
Galicia & 2.255 & 1.501 & 468 & 468 & 4.282 & 1.472 & 1.416 \\
Madrid & 3.684 & 1.979 & 815 & 816 & 7.830 & 2.671 & 2.865 \\
Murcia & 742 & 800 & 164 & 164 & 1.639 & 562 & 615 \\
Navarra & 407 & 800 & 84 & 84 & 833 & 286 & 288 \\
Pais Vasco & 1.655 & 1.489 & 356 & 356 & 3.284 & 1.116 & 1.089 \\
La Rioja & 210 & 797 & 42 & 44 & 413 & 140 & 147 \\
\hline N & $\mathbf{2 9 . 6 4 7}$ & $\mathbf{2 0 . 7 3 3}$ & $\mathbf{6 . 3 9 6}$ & $\mathbf{6 . 3 9 6}$ & $\mathbf{6 0 . 6 6 6}$ & $\mathbf{2 0 . 7 4 8}$ & $\mathbf{2 1 . 2 4 0}$ \\
\hline
\end{tabular}


Se puede demostrar (McDowell, 2003; Jiménez et al., 2002) que el logaritmo de la función de verosimilitud conjunta es la suma de los logaritmos de las verosimilitudes de los dos modelos por separado, es decir

$$
\ln L=\ln \left\{L_{1}\left(F_{j}\right)\right\}+\ln \left\{L_{2}\left(B_{j}\right)\right\}
$$

Lo que implica que, sin pérdida alguna de información, se puede ajustar un modelo de dos partes estimando primero los parámetros del modelo de probabilidad binomial y después los del modelo de recuento truncado.

En este modelo de valla, se estima, en primer lugar, la probabilidad de acceso que dependerá de: a) las condiciones personales $\left(X_{i}\right)$ del individuo $i$ (en nuestro caso: edad, género, autovaloración del estado de salud y número de enfermedades crónicas de que ha sido diagnosticado); y b) la CA $\left(R_{i}\right)$ en la que reside. Esta primera parte debe estimarse con una especificación que utilice una función de probabilidad binomial. De entre el amplio espectro posible, se ha seleccionado la función logística por su versatilidad y por la relación tan estrecha entre los parámetros estimados y la probabilidad de uso. Si decimos que $Y_{j}$ sigue una función binomial, entonces la probabilidad de que se acuda a un servicio sanitario $j$, es decir la probabilidad de que $y_{j}=1$ se suele presentar bajo la siguiente notación:

$$
P\left(Y_{j}=1 \mid X_{i}, R_{i}\right)=\frac{1}{1+\exp -\left(\beta_{j} X_{i}\right)}
$$

En segundo lugar se estima, en segundo lugar la probabilidad de los siguientes usos $-F_{j}\left(R_{i} ; X_{i} ; B_{j}\left(R_{k} ; X_{i}\right)\right)$ - que dependerá de: a) que el individuo haya accedido al siste$\mathrm{ma}$; b) las condiciones personales de este individuo $i$; y c) la CA en que viva. Formalmente, debe utilizarse un modelo de recuento truncado. Un modelo de recuento es un modelo de regresión en el que la variable dependiente solo puede adoptar números naturales $(y \in N:\{0,1,2,3 \ldots\})$. Un modelo de recuento truncado es aquel en el que se excluye la posibilidad de que se produzcan algunos valores. En nuestro caso, el 0 no es un valor posible en esta segunda parte, puesto que todos los pacientes ya han decidido «pasar la valla» y acudir, por lo menos una vez al servicio sanitario. Existen dos posibilidades para modelar este proceso: a) mediante regresión de poisson truncada; y b) mediante regresión binomial negativa truncada. La diferencia entre ambas consiste en que la regresión de poisson se encuentra sometida a una restricción que consiste en que el valor medio y la desviación estándar de la función relacional de la muestra ha de ser la misma, restricción que desaparece en la regresión binomial negativa en la que la desviación estándar puede ser distinta de la media. Esta distinta dispersión puede, a su vez, modelizarse de dos formas distintas, como una función de la media esperada y como una cantidad constante.

En este trabajo se han realizado las tres anteriores estimaciones seleccionando, en cada caso, las estimaciones poisson o binomial negativa en función de la significa- 
ción del parámetro que modeliza la sobredispersión y discriminando entre las dos modelizaciones de la función binomial-negativa en función de la bondad del ajuste medido por la reducción de la función de verosimilitud. Si $Y_{j}$ sigue una distribución de Poisson de media (y desviación típica) $\lambda_{j}$, se dice que la probabilidad de $y_{j}$ consumos del servicio sanitario $j$, por parte del individuo $i$, es:

$$
P\left(Y_{j}=y_{i j} \mid X_{i}, R_{i}, \lambda_{j}\right)=\frac{e^{\lambda_{j}} \lambda_{j}^{y_{i j}}}{y_{i j} !}
$$

Donde $\lambda_{j}=\exp \left(X_{i} \beta\right)$. Por su parte la función binomial-negativa se puede representar como una mezcla poisson-gamma, en la forma:

$$
P\left(Y_{j}=y_{i j} \mid X_{i}, R_{i}, \alpha_{j}\right)=\frac{e^{-v_{j} \exp \left(X_{i} \beta_{j}\right)}\left(-v_{j} \exp \left(X_{i} \beta_{i}\right)\right)^{i j}}{\Gamma\left(y_{i j}+1\right)}
$$

Donde $v_{j}$ es un parámetro desconocido que sigue una distribución Gamma de parámetros $(1 / \alpha, \alpha)$, de media $l$ y de varianza $\alpha$. Este último es el parámetro por el que se introduce la sobredispersión.

En los modelos representados, $X_{i}$ es una matriz que contiene las variables individuales que pueden tener influencia en el consumo de servicios sanitarios, y $\mathrm{R}_{\mathrm{i}}$ es la matriz de dummies dicotómicas que recoge la CA de residencia del individuo entrevistado. Una vez estimados los modelos por máxima verosimilitud nuestros datos de interés, sin menoscabo del resto, serán las estimaciones de los parámetros asociados a las dummies regionales $\left(\beta_{R}\right)$ que por construcción, como una propiedad de todos los modelos de regresión múltiple, nos indicaran la influencia de residir en cada CA para recibir un determinado servicio estandarizado por el resto de características personales. Dicha influencia puede transformase en Odds Ratio o en ratios de incidencia con unas sencillas operaciones algebraicas. Si $\beta_{l, j, R}$ es, en el modelo logístico, la estimación del parámetro asociado a la CA $R$ en el servicio sanitario $j$, y $\beta_{2, j, R}$ es lo mismo pero en el modelo de recuento, los odds, ratio (OR) y los ratios de incidencia (incidence rate ratios: IRR) se obtienen:

$$
\begin{aligned}
& O R_{j R}=\exp \left(\hat{\beta}_{1 j R}\right) \\
& I R R_{j R}=\exp \left(\hat{\beta}_{2 j R}\right)
\end{aligned}
$$

La estimación de este tipo de modelos obliga a definir un individuo de referencia, respecto al cual se estiman la probabilidad relativa de uso y los ratios de incidencia. En nuestro caso, el individuo de referencia es una mujer, residente en Madrid, de edad media, que goza de buena salud y que no ha sido diagnosticada de ninguna enfermedad crónica. La elección de dicho individuo se debe a que esas son las características más usuales de la muestra, aunque, por otro lado, la elección de uno u otro in- 
dividuo de referencia no afectan a las relaciones de probabilidad de uso entre dos $\mathrm{CCAA}^{9}$ que son nuestros datos de interés.

Una vez obtenidos los OR's de uso se calcula, para cada año y servicio sanitario, la dispersión de los mismos así como la existencia o no de convergencia durante el período, medida como convergencia sigma $(\sigma)$. Esta se produce (Sala i Martín, 1996) cuando se reduce, de forma significativa, la desviación estandar del logaritmo de la variable de estudio a lo largo del tiempo. En nuestro contexto, implica que si durante un periodo de tiempo se reduce significativamente la dispersión en el acceso a los servicios sanitarios en las CCAA puede afirmarse que existe convergencia sigma en el acceso y vicerversa ${ }^{10}$.

Finalmente, se ha construido un panel con los OR's e IRR's de uso estimados y el gasto sanitario público territorializado por CCAA, obtenido de las estadísticas de gasto sanitario público elaboradas por el Observatorio del SNS ${ }^{11}$. Los datos de gasto público son los consolidados de todas las Administraciones públicas (AAPP), es decir, la suma de gastos e inversión sanitaria pública de todas las AAPP presentes en un territorio. Estos se han transformado en la variable $G_{i, t}$ que es el gasto sanitario público per capita relativo a Madrid. Es decir:

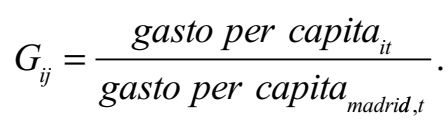

Dicha transformación lineal estacionaliza la serie y otorga a la CA de Madrid, para cada año, el valor 1 y al resto de CCAA un valor de gasto per cápita relativo respecto a Madrid, lo que permite su comparación lineal con las variables de uso (OR's e $I R R$ 's). Debido a que no se dispone de datos oficiales de gasto territorializado anteriores a 1995 el panel solo abarca las fechas comprendidas entre 1995 y 2003 . La correlación se estima mediante un modelo de regresión para datos de panel con la siguiente estructura:

$$
U S O_{j, i, t}=\alpha_{j}+\beta_{j} G_{i, t}+v_{j, i}+\varepsilon_{j, i, t}
$$

9 Por ejemplo si la probabilidad de consumir medicamentos es doble en Andalucía que en Madrid, es doble para dos individuos enfermos que consumen muchos medicamentos y también es doble para dos individuos sanos que consumen menos medicamentos. Es decir la elección de uno u otro individuo de referencia no afecta a la probabilidad relativa de cualquier CA respecto a Madrid que es lo que se ha calculado.

10 Para detectar cuando las diferencias son significativas se realiza el test de Levene (test F). Este consiste en comparar las hipótesis nula y alternativa siguientes $\left(H_{o}: \sigma_{t}^{2}=\sigma_{t+1}^{2} ; H_{o}: \sigma_{t}^{2} \neq \sigma_{t+1}^{2}\right)$. Para un determinado nivel de confianza $(0,05)$, mediante el contraste del cociente de varianzas, el test $\mathrm{F}$ nos ofrece una medida de significación estadística

${ }_{11}$ Disponibles en la sección de estadísticas de la página web http://www.msc.es. También se han ensayado correlaciones con el capital sanitario público territorializado por CCAA, obtenido de las estadísticas de capital público elaboradas por el Instituto Valenciano de Investigaciones Económicas (IVIE) y la Fundación Banco Bilbao Vizcaya Argentaria (FBBVA). Los resultados obtenidos son mucho menos significativos lo que obedece al hecho de que, a efectos del servicio sanitario público, el gasto público es mucho más relevante que el capital público. 
Donde USO, que representa las variable independiente en cada caso, es decir, los $O R$ 's o los IRR's de uso del servicio sanitario $j$ de la CA $i$ en el año $t$ estimados anteriormente, depende de $G$ que es el gasto público territorializado, y los componentes estocásticos. $v_{j, i} y \varepsilon_{j, i, t}$. Finalmente $\alpha_{j}$ y $\beta_{j}$ son los parámetros a estimar. Un valor significativo de indicará el grado de correlación entre gasto público y probabilidad de uso. Dicho modelo de regresión puede estimarse mediante un estimador consistente (de efectos fijos) o bien mediante un estimador eficiente (de efectos variables). La discriminación entre ambos se llevará a efecto mediante el test de Hausman ${ }^{12}$.

\section{Resultados}

El cuadro 3 recoge un resumen de los resultados más generales obtenidos de las regresiones logísticas utilizadas en la primera etapa del modelo de valla para cada uno de los servicios públicos analizados. Se recogen los correspondientes al primer año 1987 y el último 2003.

De los mismos se desprende un ajuste aceptable y una estimación general de todas las variables explicativas introducidas. Un gran número de dummies geográficas son altamente significativas, lo que implica que las diferencias respecto al individuo de referencia, domiciliado en Madrid, son estadísticamente significativas.

Por su parte, el cuadro 4 recoge un resumen de los resultados de los modelos de recuento truncados que han servido para estimar la segunda etapa del modelo de va1la. También se presentan sólo los resultados para 1987 y para 2003 de los cinco servicios públicos analizados. Los modelos de recuento definitivamente seleccionados (en función de la significación del parámetro que modela la sobredispersión) han sido, para dichos años el modelo de poisson truncado (ztp) y el binomial negativo truncado, con dispersión en función de la media (ztnb_media). El ajuste en general es bueno y la significación de las variables explicativas también general.

De los coeficientes estimados según los modelos de regresión anteriores se han obtenido los OR's e IRR's de uso de los servicios sanitarios públicos por CCAA y se ha procedido a comprobar la evolución de la probabilidad de uso a lo largo del tiempo en función de los modelos de convergencia sigma. El Cuadro 5 recoge los resultados.

En la columna $O R$ se representa la dispersión entre CCAA de la probabilidad de usar por primera vez un servicio. Por su parte, la columna IRR representa la dispersión entre CCAA de la probabilidad de usar repetidas veces dicho servicio. Dichas estimaciones, todas ellas absolutamente significativas, presentan un rango intercuartílico del 22,3\% al 38,5\% (mediana: 28,5 ).

12 Dicho test calcula el estadístico $H=\left(\beta_{c}-\beta_{e}\right)^{\prime}\left(V_{c}-V_{e}\right)^{-1}\left(\beta_{c}-\beta_{e}\right) ; \mathrm{H} \sim \chi_{n}^{2}$, donde: $\beta_{c}$ es el vector de estimaciones del estimador consistente; $\beta_{e}$ es el vector de estimaciones del estimador eficiente; $V_{c}$ es la matriz de covarianzas del estimador consistente; $V_{e}$ es la matriz de covarianzas del estimador eficiente; los grados de libertad de $\chi_{n}^{2}$ es el número de variables del modelo, incluida la constante. 


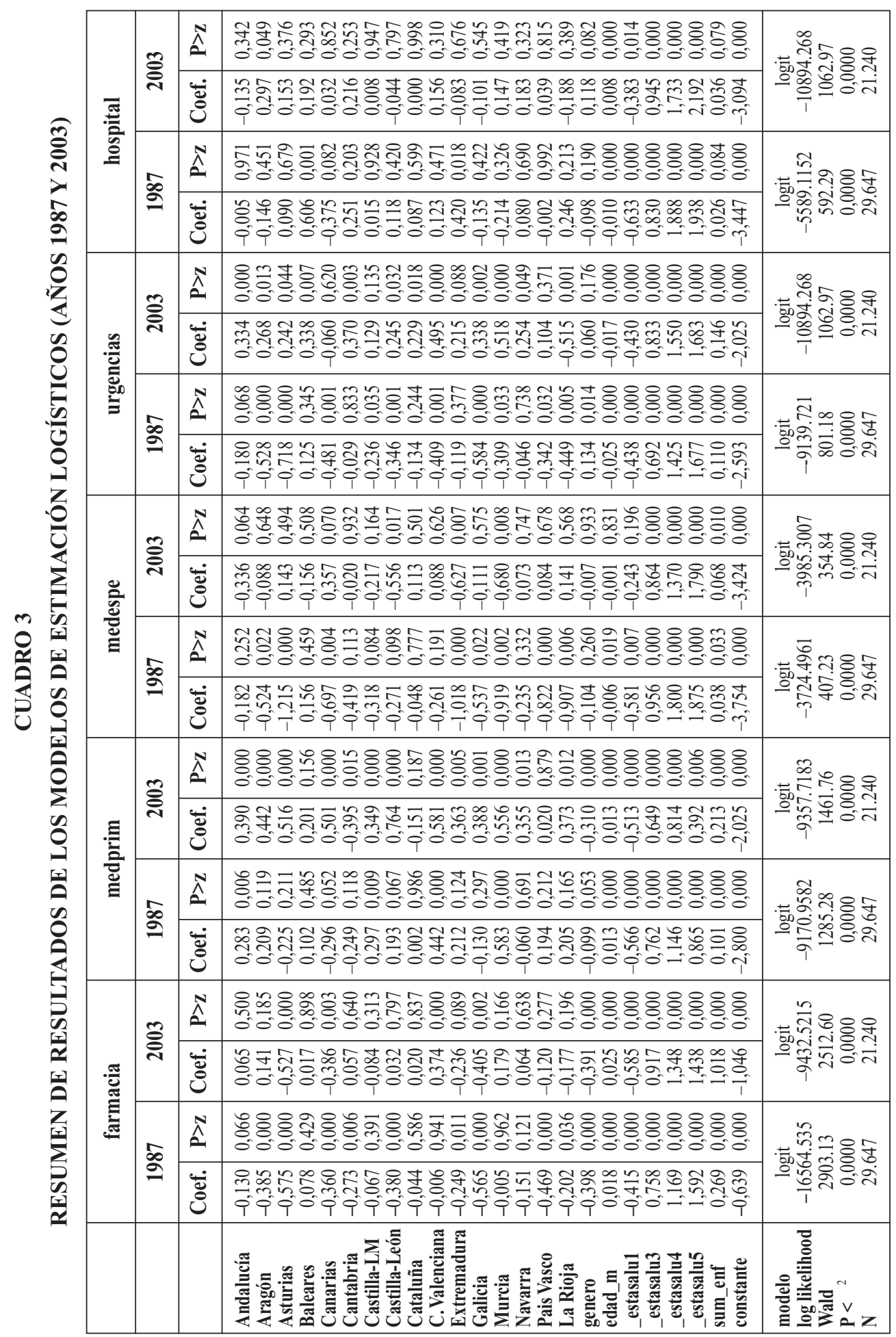




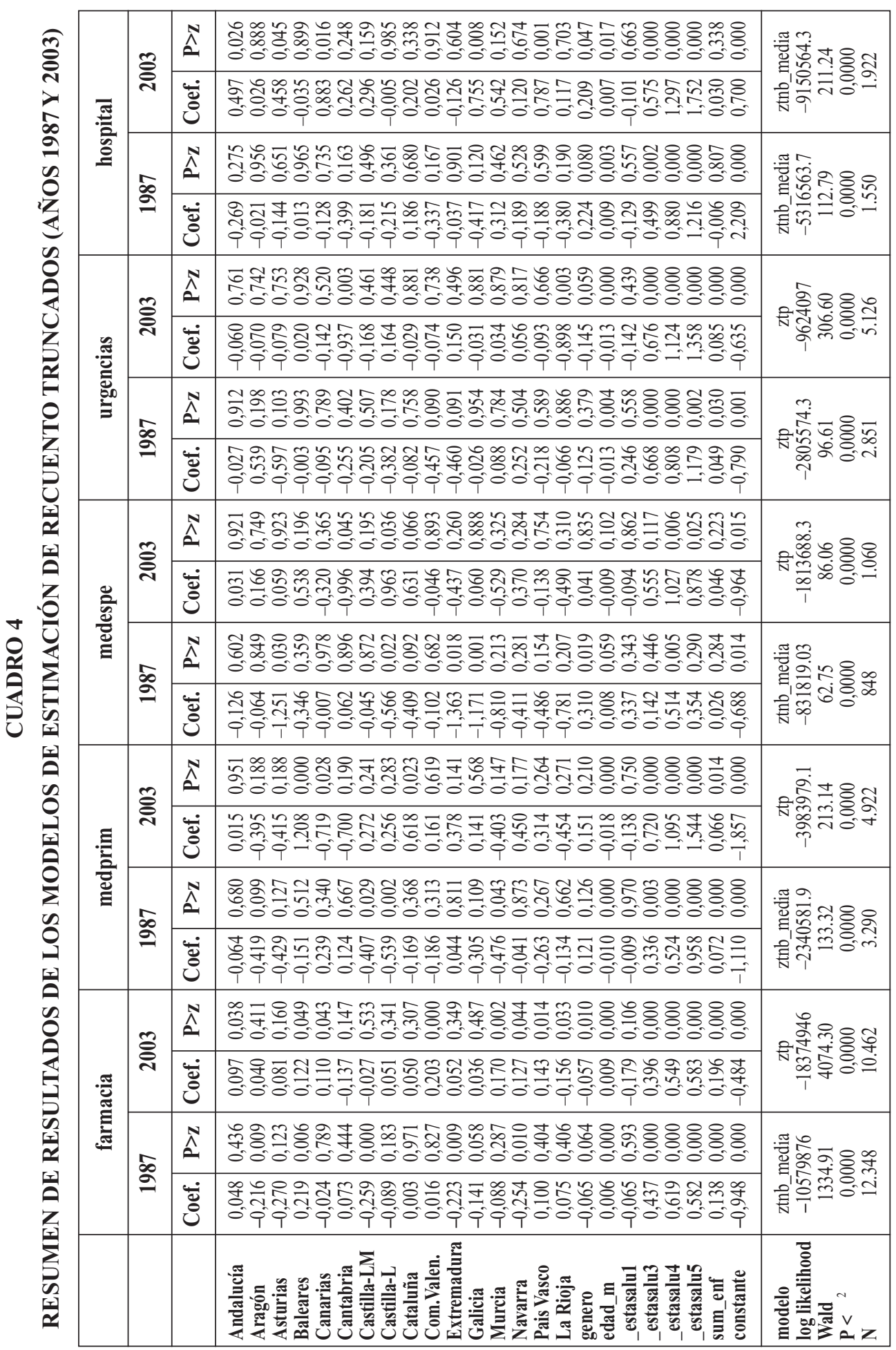




\section{CUADRO 5}

CONVERGENCIA SIGMA EN USO DE SERVICIOS SANITARIOS 1987-2003

\begin{tabular}{|c|c|c|c|c|c|c|c|c|c|c|}
\hline & \multicolumn{2}{|c|}{ farmacia } & \multicolumn{2}{c|}{ medprim } & \multicolumn{2}{c|}{ medespe } & \multicolumn{2}{c|}{ urgencias } & \multicolumn{2}{c|}{ hospital } \\
\cline { 2 - 11 } Año & OR & IRR & OR & IRR & OR & IRR & OR & IRR & OR & IRR \\
\hline $\mathbf{1 9 8 7}$ & 0,206 & 0,147 & 0,245 & 0,223 & 0,381 & 0,451 & 0,232 & 0,400 & 0,233 & 0,201 \\
$\mathbf{1 9 9 3}$ & 0,276 & 0,220 & 0,187 & 0,235 & 0,198 & 0,314 & 0,337 & 0,546 & 0,242 & 0,536 \\
$\mathbf{1 9 9 5}$ & 0,295 & 0,320 & 0,382 & 0,519 & 0,490 & 0,591 & 0,300 & 0,766 & 0,364 & 0,654 \\
$\mathbf{1 9 9 7}$ & 0,253 & 0,183 & 0,236 & n,d, & 0,425 & n,d, & 0,223 & 0,677 & 0,385 & 0,680 \\
$\mathbf{1 9 9 9}$ & 0,169 & n,d, & 0,330 & 0,223 & n,d, & n,d, & n,d, & n,d, & 0,293 & 0,172 \\
$\mathbf{2 0 0 1}$ & 0,212 & 0,141 & 0,318 & 0,306 & 0,263 & 0,348 & 0,224 & 0,483 & 0,125 & 0,221 \\
$\mathbf{2 0 0 3}$ & 0,230 & 0,097 & 0,292 & 0,509 & 0,285 & 0,471 & 0,240 & 0,468 & 0,138 & 0,317 \\
\hline
\end{tabular}

Los resultados muestran que, a igualdad de estado de salud, la probabilidad de usar un servicio sanitario puede ser muy distinta en función de la CA de residencia. Por ejemplo, en 2003 y en el caso de un primer acceso a los servicios sanitarios, la probabilidad de tomar medicamentos varía en un $\pm 23 \%$ respecto a la media, la probabilidad de visitar a un médico generalista es del $\pm 29,2 \%$, la probabilidad de visitar un médico especialista se sitúa en un $\pm 28,5 \%$, la probabilidad de acudir a un servicio de urgencias asciende al $\pm 24 \%$, y la probabilidad de internarse en un centro hospitalario oscila en un $\pm 13,8 \%$. Por su parte, la inequidad en los posteriores accesos también se mueve en parámetros similares.

- En el caso del consumo de medicamentos, se observa un incremento de la dispersión entre 1987 y 1995, incremento significativo tanto en el caso de OR's (pvalor: 0,016) como IRR's (p-valor: 0,002) que se reduce hasta 1999, fecha a partir de la cual vuelve a incrementarse la dispersión en el caso de un primer consumo de medicamentos.

- Respecto de la asistencia médica generalista, se registran suaves oscilaciones en la dispersión del primer acceso al servicio (OR), pero las más fuertes se producen en la segunda fase de la valla. La dispersión de los IRR suben en el periodo 1987-1995 (p-valor: 0,001) y en el periodo 1999-2003 (p-valor: 0,001)

- La atención por médico especialista sufre una evolución similar al anterior servicio: una primera etapa de crecimiento desde 1993-1995, significativa tanto desde el punto de vista de los OR's (p-valor: 0,000) como los IRR (p-valor: 0,008), así como un supuesto descenso desde 1995 hasta 2001, también signficativas en OR's (p-valor: 0,008) e IRR (p-valor: 0,021).

- Aunque con una evolución similar, tanto en el caso de la primera decisión de acudir como de las sucesivas, el patrón de comportamiento de la dispersión en el caso de asistencia por equipos de urgencias sólo muestra cambios significativos en el caso de los IRR's. La dispersión se incrementa durante el pe- 
EVOLUCIÓN DE LA EQUIDAD INTERTERRITORIAL EN EL ACCESO A LOS SERVICIOS... 183

FIGURA 1

\section{CONVERGENCIA SIGMA EN EL ACCESO A CINCO SERVICIOS SANITARIOS} PÚBLICOS: 1987-2003

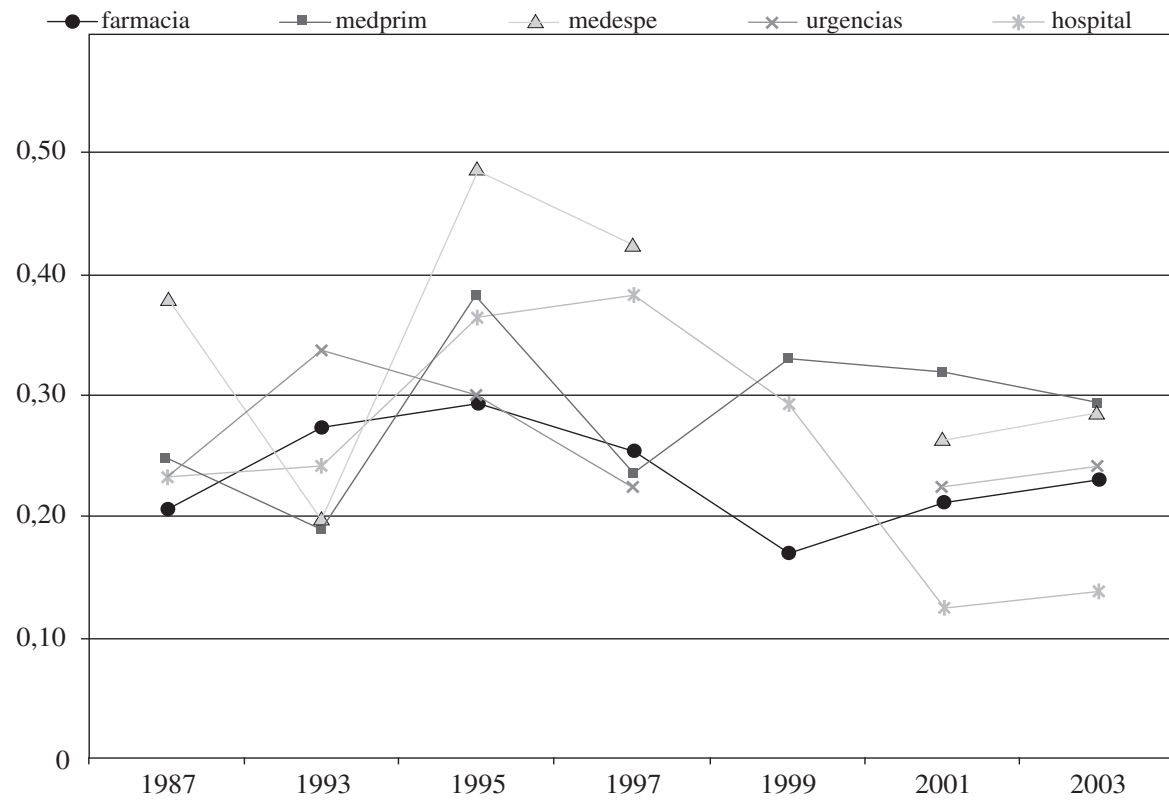

FIGURA 2

CONVERGENCIA SIGMA EN LA UTILIZACIÓN DE CINCO SERVICIOS SANITARIOS PÚBLICOS: 1987-2003

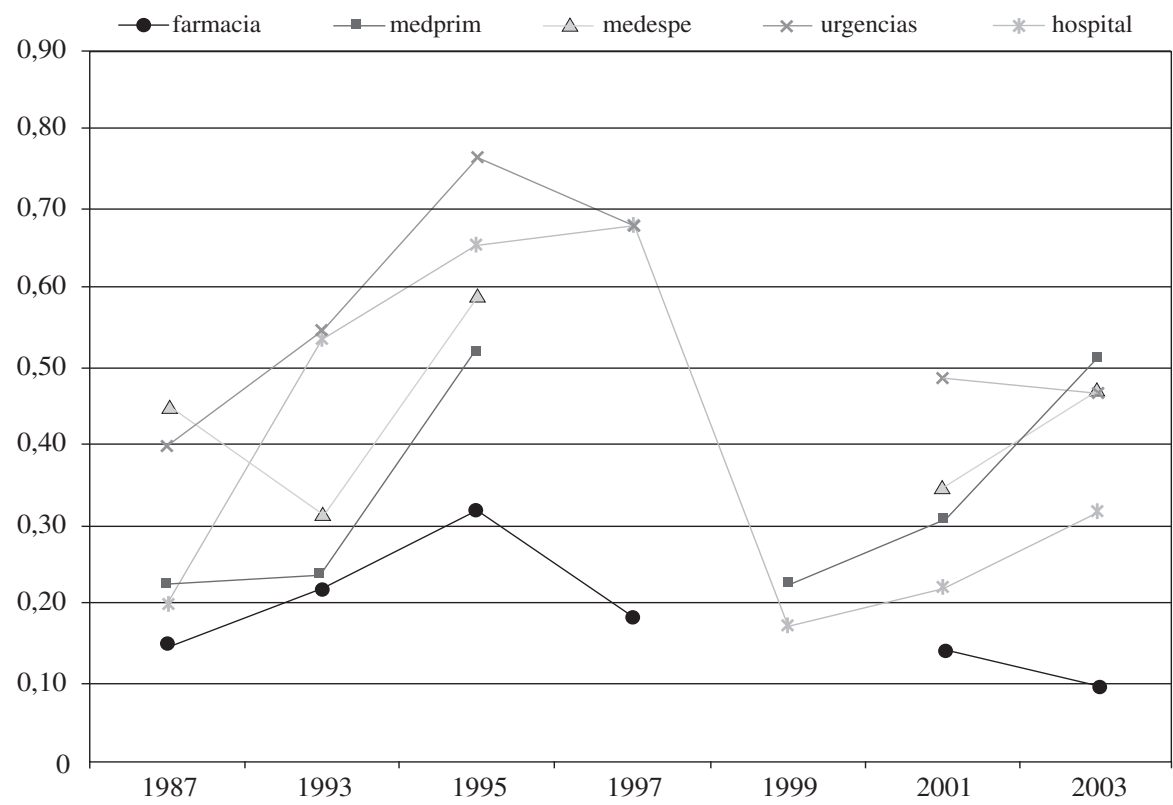


ríodo 1987-1995 (p-valor: 0,005) y disminuye durante el período 1995-2001 (p-valor: 0,024).

- En el caso de asistencia hospitalaria, para el primer acceso al servicio se produce un incremento significativo de la dispersión entre 1987 y 1997 (p-valor: 0,026) que se reduce durante el período de 1997 a 2001 ( p-valor: 0,000). Para los sucesivos accesos al sistema hospitalario las diferencias son bastante más acusadas, evidenciándose un incremento de la dispersión entre 1987 y 1997 (p-valor: 0,000) y un brusco descenso entre 1997 y 1999 (p-valor: 0,000$)$

En definitiva, se obtiene un patrón de comportamiento relativamente estable en las cinco dimensiones analizadas, de forma que, a igualdad de estado de salud, se produce un incremento de la dispersión de la probabilidad de usar un servicio sanitario en función de la CA de residencia entre el inicio de los 90 y mediados de la década. A partir de dicho momento la dispersión disminuye hasta finales de los 90 , momento en el que o bien se estabiliza o bien la dispersión tiende a volver a crecer, esta vez moderadamente. En cualquier caso, en todas las dimensiones analizadas, salvo en acceso a servicios hospitalarios, la desigualdad en el acceso en 2003 además de alta es similar a la que había en 1987. Es decir, si bien no se produce evidencia significativa de que un deterioro de la equidad en el acceso y uso de los servicios sanitarios, tampoco se aprecia ningún tipo de reducción, por lo que se hace necesario seguir controlado la evolución de dicha variable en el futuro.

Finalmente, como se ha argumentado en la introducción, interesa comprobar hasta que punto se esta produciendo una alteración en el acceso y posteriores usos de sistema sanitario condicionado por el creciente incremento y dispersión del gasto sanitario provocado, al menos parcialmente, por el proceso de descentralización de la gestión sanitaria iniciado a principios de los años ochenta del pasado siglo. En este sentido, la estimación lineal del panel elaborado con el uso y el gasto sanitario, para cada uno de los cinco servicios analizados, arroja los siguientes resultados (Cuadro 6).

Con las reservas que implica el trabajar con tan pocos grados de libertad, los resultados indican que, en lo que se refiere al primer acceso, se produce una correlación positiva y significativa en los servicios de asistencia médica general, asistencia médica especializada y servicios de urgencias respecto al gasto sanitario. No se constata dicha correlación tanto en consumo de medicamentos prescritos por el SNS como en asistencia hospitalaria. En cuanto a los sucesivos accesos al SNS, no se produce ningún tipo de correlación significativa entre la probabilidad de acceso y el gasto público en ninguna de las dimensiones de uso analizadas.

La interpretación resulta intuitiva. En aquellos servicios sanitarios en los que el paciente adopta por sí sólo la decisión de acceder al sistema (asistencia por generalista, especialista o urgencias) se produce una estrecha correlación entre uso y gasto. Sin embargo, cuando la asistencia sanitaria tiene que estar recomendada por un facultativo entonces probabilidad de uso y gasto son independientes. Na- 


\section{CUADRO 6}

\section{CORRELACIONES SIGNIFICATIVAS ENTRE OR'S Y GASTO SANITARIO TERRITORIALIZADO POR CC.AA.: 1995-2003}

\begin{tabular}{|c|c|c|c|}
\hline & medprim & medespe & urgencias \\
\cline { 2 - 4 } & OR & OR & OR \\
\hline coef. & 2,8769 & 0,7791 & 2,3930 \\
p-valor & 0,000 & 0,011 & 0,000 \\
modelo & fijos & variables & fijos \\
F/Wald & 31,38 & 6,42 & 47,32 \\
$\mathbf{P}$ & 0,0000 & 0,0113 & 0,0000 \\
$\mathbf{R}^{2}$ intra & 0,3190 & 0,0339 & 0,4139 \\
$\mathbf{R}^{2}$ entre & 0,0914 & 0,2319 & 0,0037 \\
$\mathbf{R}^{2}$ total & 0,0321 & 0,1167 & 0,0878 \\
$\mathbf{N}$ & 85 & 85 & 85 \\
grupos & 17 & 17 & 17 \\
\hline
\end{tabular}

die puede consumir medicamentos con receta o ingresar en régimen de internado hospitalario sin prescripción facultativa. Por supuesto que ulteriores visitas, consumos de medicamentos o estancias hospitalarias también deben ser prescritas por el facultativo.

El resultado anterior pone de manifiesto que, en el caso español, la heterogeneidad en la práctica médica no está condicionada por la oferta o el gasto sanitario. Es decir, a igualdad de estado de salud distintos facultativos pueden prescribir tratamientos distintos, pero generalmente su decisión no se basa en la cantidad de servicio o en el gasto público de su CA. Sin embargo, las decisiones de los pacientes sí se basan, generalmente, en la oferta de servicios o en el gasto público de su CA. De forma que, por ejemplo, a igualdad de estado de salud, en las CCAA donde existen unos servicios de urgencias mejor atendidas o más y más ágiles consultas tanto de generalistas como de especialistas, el acceso es mayor.

\section{Conclusiones}

La equidad como objetivo ético social ha adquirido en las últimas décadas una importancia central en la configuración de la actividad del sector público de los países más desarrollados. En España, la equidad interterritorial constituye una de las mayores preocupaciones del ordenamiento jurídico en general y de los objetivos de la LGS en particular. La profundización, en un sistema descentralizado de gestión de servicios públicos fundamentales (como el de sanidad) genera un especial interés en la observación de la evolución de algunas variables de igualdad interterritorial, muy es- 
pecialmente en la equidad de acceso al servicio sanitario independientemente de la CA de residencia.

Preocupa especialmente que la descentralización sanitaria esté provocando un crecimiento desordenado del gasto por parte de las CCAA y, más preocupante desde el punto de vista de la equidad, que un crecimiento de la heterogeneidad en el gasto pueda llegar a provocar inequidad en el acceso en función de la CA de residencia.

El objetivo de este trabajo ha sido el de medir la probabilidad de acceso estandarizada de un individuo a los servicios sanitarios públicos en cada CA. La estandarización se ha realizado de forma indirecta y teniendo en cuenta la edad, género e indicadores objetivos (enfermedades diagnosticadas) y subjetivos (autovaloración) del estado de salud. El método de estandarización ha sido el de regresión de un modelo en dos partes, una primera estimada mediante regresión logística y una segunda estimada mediante regresión de de recuento truncada.

Los resultados muestran que, a igualdad de estado de salud, la probabilidad de usar un servicio sanitario es muy distinta en función de la CA de residencia. Las mayores diferencias se suelen producir en la asistencia a la consulta médica. A igualdad de estado de salud y sólo en función de la CA de residencia, en 2003 la dispersión en la asistencia a consulta médica primaria fue de un $29,2 \%$ respecto a la media. La menor dispersión se produjo en el acceso a los servicios hospitalarios donde fue del $13,8 \%$ respecto a la media.

Si la situación para el año 2003 es de una considerable divergencia, respecto a la evolución en el periodo 1987-2003, no puede admitirse de ninguna caso la hipótesis de convergencia durante los 17 años considerados. Para todos los servicios sanitarios considerados, salvo el caso de un primer acceso a los servicios hospitalarios, nos encontramos en 2003 con una inequidad en el acceso similar a la que había en 1987. También puede apreciarse, aunque en unos servicios de una forma más acentuada que en otros, la siguiente evolución temporal de la inequidad. Esta parece crecer, desde el corte de 1987 hasta mediados de los años 90. A partir de dicha época vuelve a descender hasta finales de los 90 situándose en valores similares a los originales. Finalmente, desde 1999 hasta el corte de 2003, la inequidad o se mantiene constante o tiende a crecer.

El hecho de que se produzca una inequidad en el acceso a los servicios sanitarios no implica que esta sea debida a una desigualdad del gasto público en sanidad, sólo porque ambas coincidan en el tiempo. La tercera parte de la investigación trata de correlacionar ambas variables elaborando un panel de datos de uso sanitario y gasto sanitario publico de las 17 CCAA durante los años de las encuestas.

Los resultados, que no obstante deben ser considerados con cautela por la falta de grados de libertad, muestran que efectivamente el uso de los servicios sanitarios públicos en cada CA está correlacionado con el gasto y con el capital sanitario público de dicha CA, pero sólo en aquellos servicios cuya decisión de acceso depende exclusivamente del usuario. Es decir, cuando la decisión de acceso es una decisión unilateral de paciente (como en el caso de acudir a un médico generalista, un especialista 
o un servicio de urgencias) un incremento del gasto por parte de una CA, provoca un mayor acceso por parte de los usuarios residentes en dicha CA y viceversa, pero cuando la decisión del acceso debe estar prescrita por un facultativo (caso de la emisión de una receta, internamiento en un centro hospitalario o sucesivas visitas) el incremento de gasto de una CA no influye en las decisiones de los facultativos, lo cual puede ser muestra de que, al menos de momento, la variabilidad de la práctica clínica no está motivada por la política de gasto de cada CA.

Los resultados, no obstante todos los problemas metodológicos apuntados en el texto, muestran la necesidad de monitorizar la inequidad territorial en el acceso y de, en la medida de lo posible, mejorar la medición mediante la incorporación de los datos de la encuesta de 2005 (de próxima publicación) y más desagregados. El hecho de que, hasta 2003, la variabilidad de la práctica clínica no haya estado correlacionada por las políticas de gasto regionales es un dato esperanzador, pero no implica que no lo vaya a estar en el futuro. Por su parte tampoco debe olvidarse la correlación detectada entre inequidad en el acceso y desigualdad en el gasto sanitario público por CCAA (cuando la decisión es tomada unilateralmente por parte del paciente) sobre todo porque ambas magnitudes han tendido a crecer desde 1999.

\section{Bibliografía}

[1] ASESINA, A; DANNIGER, S. y ROSTANO, M. (1999): Redistribution Through Public Employment: The Case of Italy; IMF working paper, N. ${ }^{\circ}$ 17; Washington, D.C.

[2] ALVAREZ, B. (2001): «La demanda atendida de consultas médicas y servicios urgentes en España». Investigaciones Económicas. XXV (1). 93-138.

[3] ATKINSON, A.B. y STIGLITZ, J.E. (1988): Lecciones sobre economía pública, Instituto de Estudios Fiscales, Ministerio de Economía y Hacienda, Madrid.

[4] BENACH, J., YASUI, Y. (1999). «Geographical patterns of excess mortality in Spain explained by two indices of deprivation». Journal of epidemiology and community health. 53 (7). 423-431.

[5] BIRCH, S. ABELSON, J (1993): «Is reasonable access what we want? Implications of, and challenges to, current Canadian policy on equity health care» International Journal of Health Services. 23. 629-653.

[6] BORELL, C. REGIDOR, E. ARIAS, L.C. NAVARRO, P. PUIGPINÓS, R. DOMINGUEZ, V. PLASENCIA, A. (1999): «Inequalities in mortality according to educational level in two large southern european cities». International journal of epidemiology. 28. 58-63.

[7] BUCHANAN, J. (1975). «The Samaritan's Dilemma.» en E. Phelps (ed.), Altruism, Morality and Economic Theory. Russel Sage Foundation. New York.

[8] CABASES, J. (ed) et al (2007): La financiación del gasto sanitario desde la perspectiva de la experiencia comparada. Fundación BBVA. Bilbao

[9] CAMERON, A.C. TRIVEDI, P.K. (1998): Regression analysis of count data. Cambridge university press. 
[10] CULYER, A.J. VAN DOORSLAER, E. y WAGSTAFF, A. (1992a): «Comment: Utilisation as a measure of equity by Mooney, Hall, Donaldson and Gerard», Journal of Health Economics. 11. 93-98.

[11] CULYER, A.J. VAN DOORSLAER, E. y WAGSTAFF, A. (1992b): «Access utilisation and equity: A further comment». Journal of Health Economics. 11. 207-210.

[12] GODDART, M. SMITH, P. (2001): «Equity of access to health care services: theory and evidence». Social Science and Medicine. 53 (9). 1149-1162.

[13] HARSANYI, J.C. (1955): «Cardinal welfare, individualistic ethics and interpersonal comparisons of utility». Journal of Political Economy 63, 309-321.

[14] HAYEK, F.A. (1976): Law Legislation and Liberty, volumen 2, University of Chicago Press.

[15] HAYNES, R. (1991): «Inequalities in health and health service use: evidence from the General Household Survey». Social Science and Medicine. 33 (4). 361-368.

[16] Instituto de Estudios Fiscales (2005): Informe para el análisis del gasto sanitario. Ministerio de Economía y Hacienda.

[17] JHA, R. (1998): Modern Public Economics, Routledge, London

[18] JIMÉNEZ MARTÍN, S., LABEAGA J. M., MARTINEZ-GRANADO, M. (2002) «Latent class versus two-part models in the demand for physician services across the European Union». Health Economics. 11. 301-321.

[19] JIMÉNEZ MARTÍN, S., LABEAGA J. M., MARTINEZ-GRANADO, M. (2002) «Latent class versus two-part models in the demand for physician services across the European Union». Health Economics. 11.301-321.

[20] KAKWANI, N. WAGSTAFF, A. VAN DOORSLAER, E. (1997): «Socioeconomic inequalities in health: Measurement, computation and statistical inference». Journal of Econometrics. 77. 87-103.

[21] LINDBECK, A. y WEIBULL, J. (1988). «Altruism and Time Consistency: The Economics of Fait Accompli». Journal of Political Economy. 96(6). 1165-1182.

[22] LÓPEZ CASASNOVAS, G. (1998): «Financiación Autonómica y gasto sanitario público en España». Papeles de Economía Española. 76. 2-14

[23] LÓPEZ LABORDA, J. RODRIGO SAUCO, F. (2000): La cuantificación de las necesidades de gasto de las Comunidades Autónomas: Descripción y valoración de la experiencia comparada y de la investigación aplicada. Instituto de Estudios Fiscales. Madrid

[24] MCDOWELL (2003): «From the help desk: hurdle models». The Stata Journal. 3 (2). $178-184$

[25] MOONEY, G. (1983): «Equity in health care: Confronting the confusion». Effective Health Care. 1. 179-185.

[26] MOONEY, G. HALL, J. DONALDSON, C. y GERARD, K. (1991a): «Utilisation as a measure of equity: Weighing heat?. Journal of Health Economics. 10. 475480.

[27] MOONEY, G. HALL, J. DONALDSON, C. y GERARD, K. (1991b): «Reweighing heat: Response to Culyer, van Doorslaer and Wagstaff«. Journal of Health Economics. 11. 199-205.

[28] MUSGRAVE, ROBERT A. (1959): The Theory of Public Finance: a Study in Political Economy. Mcgraw-Hill. New York.

[29] MYLES, G. D. (1995): Public economics, Cambridge University Press, Cambridge. 
[30] NOZICK, R. (1974): Anarchy, State and Utopia, Basic Books. Nueva York.

[31] PEDERSEN, L.R (2001): «The Samaritan's Dilemma and the Effectiveness of Development Aid». International Tax and Public Finance. 8. 693-703.

[32] RAWLS, J. (1971): A theory of justice. Harvard University Press. Cambridge.

[33] RICE, N. y SMITH, P.C. (2001): «Ethics and geographical equity in health care». Journal of Medical Ethics, 27(4), 256-261.

[34] ROSENBERG, M.W. WILSON, K. (2000): «Gender, poverty and location: How much difference do they make in the geography of health inequalities?» Social Science \& Medicine. 51 (2). 275-287.

[35] SALA I MARTÍN, X. (1996). «The classical approach to convergence analysis». The economic journal. 106. 1019-1036.

[36] SÁNCHEZ MALDONADO, J. GÓMEZ SALA, J.S. (1998): «La financiación territorial de la sanidad: especial referencia a España». Papeles de Economía Española. 76. 19-48.

[37] SANDEL, M.J. (1982): Liberalism and the Limits of Justice, Cambridge University Press.

[38] STERN, J. (1983): «The relationship between unemployment, morbidity and mortality in Britain». Population Studies. 37. 61-74.

[39] TANZI, V. (2000): El papel del Estado y la calidad del sector público, Revista de la CEPAL, N. ${ }^{\circ} 71$.

[40] URBANOS GARRIDO, R. (2000): «La prestación de los servicios sanitarios públicos en España: cálculo y análisis de la equidad horizontal interpersonal para el período 1987-1995». Hacienda Pública Española. 153. 139-159.

[41] WATT, J. FRANKS, A. SHELDON, T. (1994): «Health and healthcare of rural populations in the UK- is it better or worse? Journal of epidemiology and Community Health. 48. 16-21. 
